\title{
Irreversible cardiac failure with intraventricular thrombosis: A novel technique of paracorporeal biventricular assist device implantation with ventricles excision
} \author{
Padova, Italy \\ From the Cardiac Surgery Unit, Department of Cardiac, Thoracic and Vascular Sciences, University of Padova, \\ Padova, Italy. \\ Disclosures: Authors have nothing to disclose with regard to commercial support. \\ Received for publication Aug 29, 2017; revisions received Oct 24, 2017; accepted for publication Nov 24, 2017; \\ available ahead of print Jan 17, 2018. \\ Address for reprints: Massimiliano Carrozzini, MD, U.O.C. Cardiochirurgia, Policlinico Universitario, Via Gius- \\ tiniani 2, 35128 Padova, Italy (E-mail: massimiliano.carrozzini@gmail.com). \\ J Thorac Cardiovasc Surg 2018;155:1632-4 \\ $0022-5223 / \$ 36.00$ \\ Copyright $\odot 2017$ by The American Association for Thoracic Surgery \\ https://doi.org/10.1016/j.jtcvs.2017.11.085
}

Massimiliano Carrozzini, MD, Francesca Toto, MD, Gino Gerosa, MD, and Tomaso Bottio, MD, PhD,

$\checkmark$ Video clip is available online.

A 63-year-old man was referred to our institution after an out-of-hospital cardiac arrest irresponsive to cardiopulmonary resuscitation, under the Lucas Chest Compression System (Physio-Control, Inc, Redmond, Wash).

\section{CASE SUMMARY}

On hospital arrival, the patient underwent extracorporeal membrane oxygenation (ECMO). Catheterization demonstrated 3-vessel coronary disease, not susceptible to revascularization because of diffuse atherosclerosis and advanced cardiac dysfunction. Transesophageal echocardiography showed extensive intraventricular thrombosis, precluding the insertion of a left ventricle vent. Although the patient was on full ECMO support, pulmonary edema and renal failure developed, with increased lactate. Cerebral computed tomography scan excluded injury, anesthesia was discontinued, and the patient awoke with preserved motor and sensory function. We managed this case with the implantation of a novel paracorporeal biventricular assist device (BiVAD), consisting of ventricles excision and connection to the atria and great arteries (Video 1).

\section{SURGICAL TECHNIQUE}

The percutaneous femoro-femoral ECMO was shifted to cardiopulmonary bypass. After aortotomy, we detected ascending aorta and aortic valve thrombosis, and a backward flushing was performed for clot removal. The great arteries were transected above the valvular level, and the whole ventricles were removed. The mitral and tricuspid leaflets were folded with a running suture. The right atrium was closed with a pericardial patch. The pulmonary artery was incised, inspected, and reconstructed with a pericardial patch. After excluding thrombosis, left atrial cannulation was achieved with an adaptive use of the sewing ring of the CentriMag 34F drainage-cannula (Abbott Laboratories, Chicago, Ill) that was secured with everting pledgeted mattress sutures. A vascular graft with side branches was interposed to connect the great arteries to the BiVAD (Figure 1). After deairing and tunneling into the mediastinum, the left ventricular assist device (LVAD) inflow cannula was secured to the left atrial cuff, and the LVAD and right ventricular assist device (RVAD) outflow cannulae were connected to the side branch of the aortic and pulmonary graft, respectively. To avoid potential systemic embolization, because of residual clots, we interposed the oxygenator in the LVAD outflow. The shift from cardiopulmonary bypass to BiVAD was completed connecting the RVAD inflow to the femoral venous cannula. The sternum was closed.

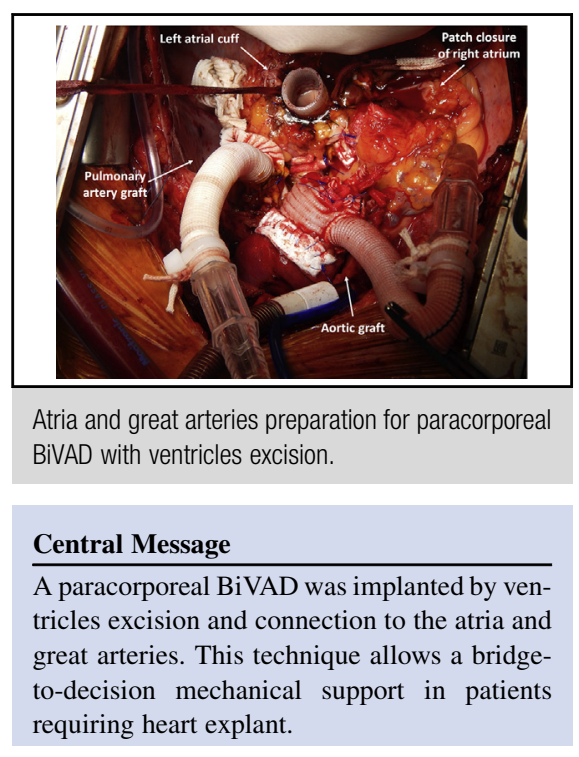

See Editorial Commentary page 1635. 


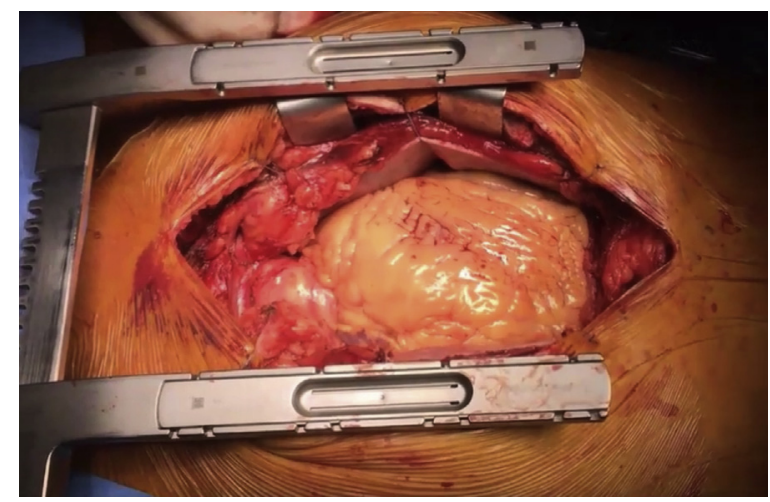

VIDEO 1. Surgical procedure. Video available at: http://www.jtcvsonline. org/article/S0022-5223(17)32845-3/fulltext.

LVAD and RVAD flow remained stable at 4.5 to $5 \mathrm{~L} / \mathrm{min}$ and 4 to $4.5 \mathrm{~L} / \mathrm{min}$, respectively. Continuous intravenous unfractionated heparin was administered for anticoagulation, monitoring activated partial thromboplastin time (target 5060 seconds) and thromboelastometry. Figure 2 shows the blood flow through the left atrial cuff by computed tomography angiography. During BiVAD support, the patient showed progressive hemodynamic stabilization with normalization of blood lactate and resolution of pulmonary edema. The patient's renal function was restored after 5 days of continuous renal replacement therapy. Unfortunately, the patient experienced a large postoperative ischemic stroke and died after 8 days of support.

\section{DISCUSSION}

Postinfarction cardiac arrest is a leading cause of death worldwide. ${ }^{1}$ ECMO is often required to stabilize the patient; however, in the absence of adequate left ventricle selfunloading, pulmonary edema occurs, mandating a shift to other mechanical supports. ${ }^{2,3}$ Intracardiac thrombosis is not rare in these situations. ${ }^{4}$ The case reported showed

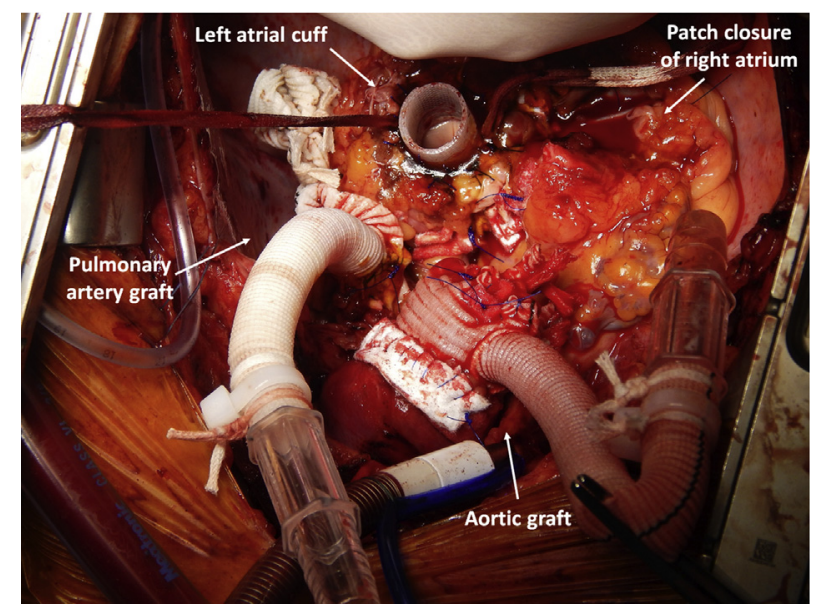

FIGURE 1. Atria and great arteries preparation for paracorporeal BiVAD implantation with ventricles excision.

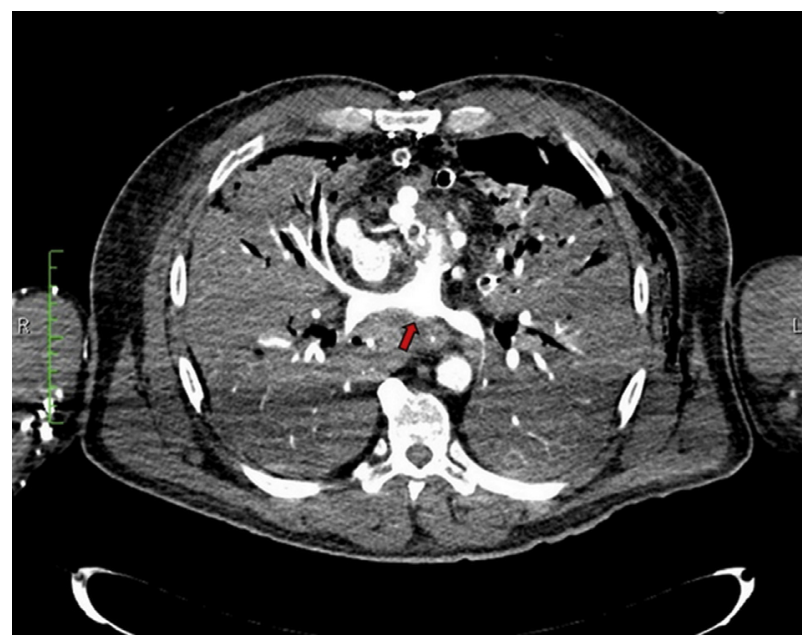

FIGURE 2. Blood flow through the left atrial cuff (red arrow), by computed tomography angiography.

irreversible biventricular failure associated with extensive intraventricular thrombosis, making heart explant the only option for survival. The thromboembolic risk, the need for end-organ function restoration, and the high likelihood of pulmonary hypertension (due to pulmonary edema and potential pulmonary embolism) contraindicated heart transplant. In this scenario, the novel paracorporeal BiVAD designed allowed an effective and less-expensive bridge-to-decision mechanical support, preferable to a high-risk total artificial heart. The BiVAD procedure itself should not be considered the primary cause of the cerebral outcome reported, because this was most likely due to a residual intra-aortic thrombosis. On the other hand, the configuration proposed could have prevented embolization from the cardiac chambers because of the oxygenator in the LVAD outflow.

Connection to the left atrium was a surgical challenge: With adaptive use of the CentriMag drainage-cannula sewing ring, we created a rigid cuff that prevented LVAD suction events. To stabilize the atria, a careful preservation of the cardiac fibrous skeleton is essential during ventricles excision. By incorporating the valve leaflets in the atrial cuff, we exploited their fibrous structure to reinforce the suture; moreover, by folding them, we covered the ventricular muscle remnants, improving hemostasis.

To manage situations in which heart preservation is deleterious (hyperacute rejection) or impossible (uncontrollable cardiac bleeding), Marinakis and colleagues ${ }^{5}$ proposed modified ECMO support with ventricles excision, atrial septectomy and anastomosis over caval axis, and right internal thoracic artery to pulmonary artery. ${ }^{5}$ This technique may have several disadvantages: difficult hemostasis, complicated heart transplant, and inadequate pulmonary perfusion. The surgical strategy we have described could apply to the same scenarios, being more simple and 
physiologic; subsequent heart transplant can be performed in the usual bicaval fashion.

A possible drawback of this support, if protracted, is the femoral venous cannulation that could limit patient mobilization; direct atrial (similar to left atrium, although with a higher risk of suction) or bicaval cannulation could represent alternative solutions.

\section{CONCLUSIONS}

Ventricles excision with connection to the atria and great arteries for BiVAD implantation proved to be a feasible bridge-to-decision mechanical support, reserved for patients requiring heart explant.

\section{References}

1. Stub D, Bernard S, Duffy SJ, Kaye DM. Post cardiac arrest syndrome: a review of therapeutic strategies. Circulation. 2011;123:1428-35.

2. Tarzia V, Bortolussi G, Bianco R, Buratto E, Bejko J, Carrozzini M, et al. Extracorporeal life support in cardiogenic shock: impact of acute versus chronic etiology on outcome. J Thorac Cardiovasc Surg. 2015; 150:333-40.

3. den Uil CA, Akin S, Jewbali LS, Dos Reis Miranda D, Brugts JJ, Constantinescu AA, et al. Short-term mechanical circulatory support as a bridge to durable left ventricular assist device implantation in refractory cardiogenic shock: a systematic review and meta-analysis. Eur J Cardiothorac Surg. 2017; 52:14-25.

4. Williams B, Bernstein W. Review of venoarterial extracorporeal membrane oxygenation and development of intracardiac thrombosis in adult cardiothoracic patients. J Extra Corpor Technol. 2016;48:162-7.

5. Marinakis S, Burki M, Abdel-Sayed S, von Segesser LK. A model of anterograde oxygenated lung blood flow in acardia. ASAIO J. 2016;62: 631-3. 